\title{
Eurostudia
}

\section{« L'histoire est là, et il faut la digérer ... il faut "la rendre conte" " : réflexions autour de la mise en fiction de l'histoire dans le roman postcolonial}

\section{Sylvère Mbondobari}

Volume 13, numéro 1-2, 2018-2019

Cultures en contact, entre régulations et représentations

URI : https://id.erudit.org/iderudit/1067286ar

DOI : https://doi.org/10.7202/1067286ar

Aller au sommaire du numéro

Éditeur(s)

Le Centre canadien d'études allemandes et européennes

ISSN

1718-8946 (numérique)

Découvrir la revue

Citer cet article

Mbondobari, S. (2018). « L'histoire est là, et il faut la digérer ... il faut "la rendre conte" » : réflexions autour de la mise en fiction de l'histoire dans le roman postcolonial. Eurostudia, 13(1-2), 319-343. https://doi.org/10.7202/1067286ar
Résumé de l'article

L’une des conséquences des lois mémorielles de 2001 et de 2005 en France fut une réflexion à propos des conditions de possibilité d'une écriture croisée de l'histoire des conquêtes coloniales en Afrique francophone. Le présent article propose dans une première partie une étude des modalités d'inscription de l'histoire coloniale dans LeRoi de Kahel et de Le Terroriste noir de Tierno Monénembo à partir des notions de " conte " et de " rendre compte » des évènements du passé. La seconde partie porte sur Madagascar, 1947 où l'écrivain malgache Jean-Luc Raharimanana mène une réflexion sur les modalités d'une réappropriation de l'histoire coloniale au nom du devoir de mémoire.
Tous droits réservés @ $@$ Le Centre canadien d'études allemandes et européennes, 2019
Ce document est protégé par la loi sur le droit d'auteur. L'utilisation des services d'Érudit (y compris la reproduction) est assujettie à sa politique d'utilisation que vous pouvez consulter en ligne.

https://apropos.erudit.org/fr/usagers/politique-dutilisation/ 
"L'histoire est là, et il faut la digérer ... il faut "la rendre conte" »: Réflexions autour de la mise en fiction de l'histoire dans le roman postcolonial

\section{Sylvère Mbondobari}

Université Omar Bongo de Libreville

\section{Résumé}

L'une des conséquences des lois mémorielles de 2001 et de 2005 en France fut une réflexion à propos des conditions de possibilité d'une écriture croisée de l'histoire des conquêtes coloniales en Afrique francophone. Le présent article propose dans une première partie une étude des modalités d'inscription de l'histoire coloniale dans Le Roi de Kahel et de Le Terroriste noir de Tierno Monénembo à partir des notions de « conte » et de « rendre compte » des évènements du passé. La seconde partie porte sur Madagascar, 1947 où l'écrivain malgache Jean-Luc Raharimanana mène une réflexion sur les modalités d'une réappropriation de l'histoire coloniale au nom du devoir de mémoire.

\section{Abstract}

One of the consequences of memorial laws passed in 2001 and 2005 in France is a reflection on the conditions of possibility of cross-writing the history of colonial conquests in French-speaking Africa. This article proposes in its first part a study of the ways in which colonial history is inscribed in Tierno Monénembo's Le Roi de Kahel ("The King of Kahel") and Le Terroriste noir ("The Black Terrorist") based on the notions of "storytelling" and the ways in which they "account" for past events. The second part deals with Madagascar, 1947 in which 
the Malagasy writer Jean-Luc Raharimanana reflects on conditions for a re-appropriation of colonial history in the name of a duty of remembrance.

Rendre l'Histoire « conte », tel est le sens que Tierno Monénembo assigne à la littérature francophone contemporaine. Dans la perspective qui est celle de l'auteur franco-guinéen, "rendre conte » signifie au moins trois processus complémentaires. Le premier suppose un retour sur l'événement historique en donnant une nouvelle vie aux faits : l'évènement historique devient un matériau brut, qui produit des images et des discours et qui peut être réactivé à tout moment. Le second souligne le processus de transformation et de passage de la réalité historique à la fiction romanesque, qui suppose sélection, amplification, et dramatisation. Le troisième processus renvoie à la «mémoire culturelle » (J. Assmann) et indique le mode de conservation et de transmission de l'histoire à travers les récits oraux ou, dans une tout autre perspective, l'imitation des dispositifs narratifs de l'oralité par l'écriture. A cet égard, Monénembo faisait remarquer que «pour un romancier, l'Histoire est un sujet inévitable. Ou on l'exploite, ou on la refoule, ou on la renie, ou on s'en moque. En tout cas, elle est là et elle hante et hantera toujours la littérature » (Fèvre 2017).

Si l'on rejoint la position de l'auteur, qui conçoit l'Histoire comme une «obsession » et comme une source inépuisable où le romancier puise dans les archives, la réflexion sur les enjeux de l'écriture littéraire de l'histoire et l'étude des modalités de la «mise en fiction" (Ricœur) deviennent incontournables. Comme le fait remarquer Michael Kohlhauer : «prétendre redire le passé comme tel, dans la pureté même de ce qui fut, n'est qu'une illusion » (Kohlhauer 2011 : 
45). En réalité, le choix des événements n'est jamais gratuit, leur valeur est bien révélatrice, non pas qu'ils soient à prendre au pied de la lettre, mais parce qu'ils véhiculent des vérités humaines qu'ils n'épuisent pas et qui ne les épuisent pas non plus, tout en leur donnant une dimension proprement poétique. Après tout, explique Kohlhauer : "l'image du passé ne devient visible pour nous qu'à travers l'idée que nous en avons, et le récit que nous en faisons à partir de notre propre présent » (Kohlhauer $2011: 45)$.

Né en Guinée (Conakry) en 1947, Tierno Monénembo quitta son pays pour fuir la dictature de Sékou Touré et s'installa en France à partir de 1973. En 1979, il publia son premier roman Les crapauds-brousse). Auteur de douze romans, d'une pièce de théâtre et lauréat de plusieurs prix littéraires ${ }^{1}$, Monénembo est considéré par la critique francophone comme l'un des plus grands auteurs africains. D'une vingtaine d'années plus jeune (né en 1967), Jean-Luc Raharimanana est un romancier, essayiste, poète, auteur de plusieurs pièces de théâtre et metteur en scène d'origine malgache. Il a la particularité de publier ses textes en français et en malagasy et de jouer lui-même dans la plupart de ses pièces. La question de l'écriture de l'histoire est perçue par les deux auteurs comme une urgence et une nécessité au sens où, plus d'un demi-siècle après l'accession de leur pays à l'indépendance, la réouverture des archives et la confrontation avec les événements passés devraient aboutir à repenser la relation entre l'Afrique et la France dans une perspective postcoloniale qui interroge le sens de l'histoire, l'époque et la vérité des faits et privilégie le croisement des regards (Mbembe 2013 : 239-248).

\footnotetext{
${ }^{1}$ Avec Les Ecailles du ciel (1986), il reçoit Le grand prix littéraire de l'Afrique Noire et une mention de la fondation Léopold Sédar Senghor, avec L'Aîné des orphelins (2000), le prix Tropiques, avec Le Roi de Kahel (2008), le prix Renaudot et avec Le Terroriste noir (2012) les prix du roman métis et Erckmann-Chatrian (2012), Grand prix Palatine et Ahmadou-Kourouma (2013).
} 
Lire le passé à partir de la trajectoire des protagonistes, de la mémoire individuelle et collective, telle est l'intention des deux auteurs. Dans le roman postcolonial, le recours à l'Histoire cosntitue tout un programme : Monénembo et Raharimanana examine dans le détail des réalités diverses, celles de l'esclavage, de la conquête et des résistances coloniales, des guerres d'indépendances ou de la construction de nations africaines. Pourquoi et comment le romancier postcolonial se réfère-t-il au passé? La première partie de notre réflexion propose différentes approches de l'écriture de l'histoire dans la littérature francophone d'Afrique. Dans une perspective diachronique, elle insiste sur les passages et les ruptures épistémologiques. La deuxième partie traite des modalités de construction d'une histoire croisée entre la France et l'Afrique dans les œuvres de Tierno Monénembo. La troisième partie aborde la question de l'appropriation de l'histoire coloniale chez Jean-Luc Raharimanana.

\section{Littérature francophone d'Afrique : la question de l'histoire}

Dans l'histoire des littératures francophones d'Afrique, les années 1930 constituent une époque charnière dans le rapport que la littérature entretient avec l'histoire précoloniale et postcoloniale marquées par la fin des Empires, la rencontre avec l'Europe et de profonds bouleversements sociaux, culturels et politiques. Précédée par la découverte de «l'art nègre » par les peintres du début du siècle ${ }^{2}$, la prise de parole des années 1920 par la première génération des écrivains africains (Filly Dabo Cissoko, Bakary Diallo, etc.) est un premier changement qui prend une forme définitive avec le mouvement de la Négritude, qui naît à Paris dans le sillage de l'équipe parisienne de l'Étudiant noir et de la revue Légitime défense et s'étend à Dakar et Brazzaville, les deux grandes capitales de l'Afrique-Occidentale française et de l'Afrique-Équatoriale française. Cette

\footnotetext{
${ }^{2}$ On citera à titre indicatif Picasso, Derain, Matisse pour ce qui est de la peinture et Guillaume Apollinaire, Blaise Cendras et Philippe Soupault en littérature.
} 
effervescence intellectuelle qui correspond à l'émergence d'une classe de penseurs (Léopold Sédar Senghor, David Diop, Aimé Césaire, Léon Gontran Damas, Frantz Fanon, etc.) se fonde sur une critique contredisant les discours coloniaux qui cherchaient à combler des lacunes de l'Histoire, à préciser certains événements et à opérer des glissements et des rectifications qui s'imposaient. Le discours sur le colonialisme (1950) de Césaire et Peau noire, masques blancs (1952) de Fanon apparaissent ainsi comme des textes programmatiques qui situent la relation à l'Histoire de toute une génération. L'exigence d'une nouvelle écriture de l'histoire négro-africaine et d'un renouvellement des relations centre/périphérie qui prendraient en compte des lectures et des pratiques différentes se lit à deux niveaux: d'une part, au niveau d'une transformation sociologique et géopolitique capitale après la Première Guerre mondiale, et, d'autre part, à l'émergence d'une forte conscience politique chez les intellectuels et écrivains de la Négritude, chez les Tiers-mondistes ainsi que dans une partie de l'intelligentsia française de gauche des années 1950 (Jean-Paul Sartre, René Dumont, Edmond Jouve, etc.).

La deuxième césure, et non des moindres, se manifeste dès le début des années 1960 avec, d'une part, la volonté d'écrire une histoire générale de l'Afrique à partir d'un fondement épistémologique spécifiquement africain par une équipe d'historiens menée par Joseph Ki Zerbo et Amadou-Mahtar M'Bow ; et d'autre part, la publication de nombreux textes épiques (Mofolo 1940; Niane 1960) qui mettent l'accent sur la notion de « résistance africaine » (Semujanga 2004 : 17-18). Cette approche d'une historiographie pensée à partir de l'Afrique tente une rupture épistémologique qui renouvellera aussi bien l'objet de la recherche que les méthodes et les pratiques. Il s'agit d'une approche de l'histoire de l'Afrique qui se retrouve également chez les écrivains qui, à la manière des frères Grimm, recueillent des textes oraux, les transcrivent (Malonga 1959; Boni 1962; Socé 1962) et les vulgarisent. 
La troisième période, qui commence au début des années 1970, est marquée par une forte appropriation des faits historiques de la période coloniale par les formes de représentation littéraire et médiatique (roman, cinéma, etc.). Elle procède d'une prise de conscience encore plus forte que celle des années 1920 et 1930 et correspond à une sélection « de ce qui est digne d'être mémorialisé. [...] Ce qui par sa grandeur mérite d'être retenu, médité, imité » (Rancière 2012 : 57). Cette troisième phase cumule les aspects d'une nouvelle poétique de l'histoire et le renouvellement de l'historiographie africaine. C'est dans ce sens qu'il faut entendre les études postcoloniales lorsqu'elles abordent la question de l'héritage colonial et celle de l'écriture de l'histoire au sens d'une écriture de l'histoire à rebours, une "Gegengeschichtsschreibung» (Rüsen 1982: 551). Ce qui fait la spécificité de l'écriture postcoloniale de l'histoire, c'est aussi bien l'élaboration d'un contre-discours, la prise en compte des mutations géopolitiques que la mise en perspective de ces transformations avec une poétique du texte littéraire. Andrew Smith souligne justement que

la rhétorique de la théorie postcoloniale a pour objet de célébrer la réouverture de choses réputées à jamais closes [...]. L'écrivain migrant est souvent considéré ici comme l'emblème de cette nouvelle libération, comme celui ou celle qui force les portes d'histoires verrouillées et de récits autocentrés (Smith $2006: 365$ ).

Cette troisième période est donc celle des écrivains migrants qui s'opposent à la «clôture du récit» (Ricœur) et font de la littérature francophone un lieu de questionnements de l'histoire et de réévaluation des savoirs coloniaux.

\section{Le Roi de Kahel, Le Terroriste noir : réinvestir l'histoire coloniale}

Le premier des grands romans historiques de Tierno Monénembo, Les Écailles du ciel (1986), déploie en pleine conscience les moyens de la fiction au service de l'Histoire. Sans prétendre faire œuvre d'historien, l'auteur fait revivre quelques pages parmi les plus tragiques de l'Histoire de l'Afrique. Cousin 
Samba, le protagoniste des Écailles $d u$ ciel, amorce ainsi une traversée qui permet de revivre l'arbitraire du pouvoir colonial, le désenchantement des indépendances et la dictature postcoloniale. Plus tard, dans Pelourinho, roman publié en 1995, c'est la question bien plus théorique de l'écriture de l'histoire de l'esclavage qui est abordée par le biais du personnage éponyme surnommé Escritore. Dans un récit complexifié par la polyphonie et les dialogues entre les narrateurs Leda et Innocencio se construisent progressivement les traces de cet écrivain africain que l'on nomme Africano pour signifier son origine ou Escritore pour signifier sa fonction d'écrivain. C'est justement cette réflexion à la fois théorique sur la question des origines et de la mémoire de l'esclavage et esthétique qui a fait dire à Monénembo que "les romans sont des tentatives désespérées de dompter l'Histoire avec un grand "H" et révéler ses fourvoiements par le biais de la petite histoire » (Chanda 2008). L'évocation de l'Afrique est un moyen pour faire revivre une «mémoire enfouie. Non pas avec des slogans, mais avec des romans, de la poésie, du théâtre », explique Monénembo (Fèvre 2017).

Dans Le Roi de Kahel (Prix Renaudot 2008), Monénembo choisit comme héros le vicomte Aimé Olivier de Sanderval, personnage historique qui se mit en tête, à la fin du XIXe siècle, d'édifier un royaume au Fouta-Djalon dans l'actuelle Guinée. Texte abondamment commenté par la critique, Le Roi de Kahel s'inscrit dans un processus de réception assez particulier comme l'ont démontré, chacun à sa manière, Bernard De Meyer (2011), Florence Paravy (2014) et Abdoulaye Imorou (2010). Que l'on l'inscrive dans une perspective postcoloniale (De Meyer), que l'on en fasse un « roman du consensus » (Imorou) ou que l'on s'intéresse aux procédés de réécriture (Paravy), le récit de Monénembo invite inévitablement à une redécouverte de l'imaginaire colonial du XIX siècle et, de facto, et à une réévaluation du discours sur les peuples de la région et les mœurs des Peuls (Migraine-George 2013: 32). 
A cet égard, la démonstration de De Meyer constitue un exemple éloquent qui prend tout son sens par la minutie qu'il déploie dans son analyse sociologique et postcoloniale de l'œuvre de Monénembo. L'étude de De Meyer se fonde sur l'interprétation du contexte éditorial et politique au moment de la publication de l'œuvre en France (De Meyer 2011 : 62-63). Cette interprétation est complétée par une lecture intertextuelle de l'œuvre qui permet, d'une part, une mise en relation avec d'autres œuvres de l'auteur, notamment Pelourinho (1995) et Peuls (2004) où le personnage d'Olivier de Sanderval apparaissait déjà vers la fin du récit. D'autre part, il s'agit pour De Meyer de démontrer que Le Roi de Kahel s'inscrit dans une tradition discursive que l'on retrouve déjà chez « des penseurs comme Aimé Césaire, Frantz Fanon, Édouard Glissant et Albert Memmi, ainsi que Jean-Paul Sartre et Jacques Derrida, qui prônent le droit à la formulation d'un propos divergent sur l'histoire » (De Meyer 2011 : 63). Pour De Meyer, « le retour au passé qu'opère le dernier roman de Monénembo ne supprime pas ce contexte d'émergence du roman, en particulier à cause du fait que ce passé se trouve être une des causes directes des tensions actuelles » (De Meyer 2011 : 63). Cette mise en perspective se présente comme une réévaluation voire, une révision $d u$ discours et de la mémoire coloniaux du XIXe siècle qui n'auraient retenu que des " grands héros de la colonisation que sont les Faidherbe, les Brazza, les Lyautey » (Chanda 2008). Sanderval, explique Monénembo lui-même, «n’a pas réussi à s'imposer face à l'énorme machine de guerre de l'administration coloniale » (Chanda 2008).

C'est aussi le point de vue de Paravy lorsqu'elle note que : «l'œuvre de T. Monénembo rejoint, mais dans une perspective littéraire, le réexamen critique des relations de voyage entrepris depuis 1980 par certains historiens africanistes, puis par des chercheurs en littérature » (Paravy 2014). Paravy évoque également l'importance des «significations sous-jacentes de cette réécriture postcoloniale » (Paravy 2014). Il ne s'agit pas de réfuter d'emblée l'hypothèse selon laquelle Le 
Roi de Kahel serait, comme l'a indiqué l'auteur lui-même, une réhabilitation de la figure d'Olivier de Sanderval oublié par le discours officiel ${ }^{3}$, mais de montrer qu'au-delà de cette figure se développe dans un moment de dialogue et d'échange une mise en scène de la société peule.

Dans ce complexe processus de mise en écriture, Le Roi de Kahel peut être lu à la fois comme une continuation et une suite de Peuls ou comme une variation des Écailles du ciel et de Pelourhino, puisqu'on y retrouve la question coloniale, la problématique de l'identité peule et la fictionnalisation de personnages historiques. Monénembo place la représentation de l'histoire coloniale dans sa vraie perspective, qui est celle d'une rencontre interculturelle où se lit en filigrane le désir de faire revivre le monde peul au moment de sa rencontre avec l'Occident. Avant tout, c'est ce moment de confrontation et de partage que l'auteur veut créer en ravivant un chapitre important de l'Histoire africaine. Au simple récit biographique se substitue l'idéalisation plus ou moins consciente du monde peul au moyen duquel Monénembo façonne et organise sa représentation de la société aristocratique.

Plus que la colonisation, plus qu'Olivier de Sanderval, c'est donc la société aristocratique qui, par un jeu de miroir, est représentée. On peut considérer que la grande Histoire de Sanderval se construit à partir des histoires qui scandent les aventures de l'explorateur. L'intérêt de ce roman, c'est qu'il ne parle pas de l'entreprise coloniale in abstracto, mais en suivant les traces et l'itinéraire d'un colon. Et si le colon est devenu un personnage romanesque soumis à

\footnotetext{
${ }^{3}$ Dans son entretien avec Tirthankar Chanda, Tierno Monénembo relève cet aspect en ces termes : "Il a été fait officiellement peul par l'almâmi. Il se considérait lui-même comme un seigneur peul. C'est la preuve que l'histoire coloniale n'a pas toujours été aussi manichéenne qu'on a voulu le dire : ce n'était pas toujours Blancs contre Noirs. Il y avait place, surtout au début, pour un autre projet colonial basé sur le respect et l'échange. L'histoire de Sanderval est en fait la petite histoire de la colonisation, la colonisation comme elle aurait pu être. Les romans sont des tentatives désespérées de dompter l'Histoire avec un grand "H" et révéler ses fourvoiements par le biais de la petite histoire » (Chanda 2008).
} 
l'imagination et aux caprices de l'auteur, c'est que Monénembo a beau jeu de composer un récit comme il l'entend et d'attribuer à son héros les traits de caractère qu'il lui plaît de lui donner. On voit bien qu'il a tenu à conserver les traits et les paroles de l'aventurier par soucis d'authenticité ou par simple convenance intellectuelle ${ }^{4}$. Ainsi, Olivier de Sanderval peut être historiquement situé d'après des indices internes finement sélectionnés par l'auteur : son identité bourgeoise, la dimension messianique et le projet du personnage.

Mais au-delà de ce jeu entre fiction et vérité déjà signalé par de nombreux critiques (Imoru 2010 : 1-13; De Meyer 2011 : 60-79; Migraine-George 2013 : IX-LII; Paravy 2014), la mise en perspective du quotidien avec la conquête coloniale donne une signification particulière à ce roman. Ce qui compte en définitive, ce n'est pas tant la conquête coloniale en soi, puisqu'elle n'apparaît que de manière implicite, mais la redécouverte du royaume du Fouta-Djalon à travers le double regard du narrateur omniscient et celui d'Olivier de Sanderval. Pour Monénembo, la relation interculturelle qui se noue au fil des pages entre le personnage principal et les Peuls, n'est pas dépourvue d'intérêt ethnoculturel renvoyant aux us et coutumes, elle offre un regard souvent critique de la société peule de l'époque reconstruite par ses soins en même temps qu'elle exhibe ses valeurs aristocratiques. Qu'il s'agisse des jeux du pouvoir, de la justice, de l'adultère, de fêtes ou de cérémonies religieuses, ce sont là autant d'opportunités de faire connaissance avec ce monde «insondable, sublime et inquiétant» (Monénembo 2008 : 145). La rencontre interculturelle est ici non seulement un lieu d'échanges émaillé de nombreux incidents critiques, d'incompréhensions et

\footnotetext{
${ }^{4}$ A ce sujet Tierno Monénembo explique que «l'essentiel de ce que j'ai raconté est vrai. Je suis parti des carnets de voyages de Sanderval. Mais pour les besoins de la fiction, j'ai inventé des personnages secondaires et amplifié certaines anecdotes pour obtenir une épaisseur dramatique. Par exemple, Sanderval raconte dans ses carnets qu'il avait rencontré une femme qui était tellement belle qu'il a demandé à son mari de la lui donner en mariage. Il ne dit guère plus. J'ai décrit la femme en question et imaginé une histoire d'amour entre les deux » (Chanda 2008).
} 
de quiproquos en tout genre - comme cette scène où Sanderval est présenté comme un prince français (Monénembo 2008: 55-58) -, mais le lieu de la valorisation d'un ethos peul où l'aristocratie peule est à l'honneur.

Battant en brèche la vision de Sanderval $d^{\prime}$ « un pays tout vierge avec des fleurs partout et des fruits étranges; peuplé de bêtes et de tribus éparses, joviales et pacifiques » (Monénembo 2008: 185), Monénembo relève de manière subtile qu'il s'agit d'un royaume multiséculaire avec une organisation sociale et politique, ses princes, ses aristocrates, ses mœurs (Boulège 1981 : 699-706). Le fait que Sanderval revendique son appartenance à cette société ou qu'il se sente contraint de mentir et de prétendre qu'il est un membre de l'aristocratie française n'est pas anodin. Il implique une reconnaissance de l'aristocratie locale comme égale des sociétés occidentales. En définitive, le récit reprend la biographie de Sanderval en ajoutant des détails qui complètent un peu le tableau esquissé par l'explorateur en même temps qu'il laisse apparaître la preuve implicite de la grandeur des rois du Fouta-Djalon, comme on le voit dans la présentation du cortège d'Alpha Yaya ou dans la représentation qu'il fait de l'almâmi à la fois chef politique et chef religieux (Botte 1990 : 37-51). Comme l'a si bien vu Tirthankar Chanda, le roman « raconte en filigrane le déclin et la chute de l'empire peul $»^{5}$ (Chanda 2008).

Suivant une vieille tradition de l'historiographie occidentale, l'Histoire, telle qu'elle est représentée dans le Roi de Kahel reste d'abord incarnée par la vie des princes et les événements qui scandent leur existence et que l'auteur contribue par son travail d'écriture à faire redécouvrir, à modeler et à enrichir. Les principales figures historiques aristocratiques (Pathé, Bôcar-Biro, Aguibou et Alpha Yaya), qui peuvent être historiquement situées, sont le témoignage de la

\footnotetext{
${ }^{5}$ Une note précise par exemple que «le roi de Labé sera arrêté quelques années plus tard et déporté au Dahomey puis en Mauritanie où il mourra en 1912 » (Monénembo 2008 : 323).
} 
puissance, de la richesse et du prestige de l'aristocratie peule. Le prétexte des pourparlers lorsqu'il s'agira de céder le Kahel à Sanderval, l'organisation de la prise de parole chez l'almâmi sont autant de traits qui donnent une marque particulière et prestigieuse à l'aristocratie locale, tout en évoquant avec force une «mémoire culturelle» (J. Assmann) qui la rattache à une vieille et prestigieuse tradition arabe. Cette focalisation sur l'aristocratie locale, ses modes d'élection, ses us et coutumes, structure une histoire peule visant à ne pas vanter uniquement la gloire de ses princes, mais aussi à décrire leur déclin qui, quoique raconté sous une forme pittoresque, revêt tout de même une grande valeur symbolique. Monénembo décrit avec une certaine complaisance les traits archaïques des mœurs locales, exalte la beauté de la région, relève le niveau d'instruction des princes et même des traits de leur personnalité en évoquant le poulâkou comme une sorte de codex qui régit la société :

Chez les Peuls, on s'assoit toujours de biais, mange de biais, parle de biais, s'allie de biais, se fait la guerre de biais et se réconcilie de biais. Se montrer franc est un manque de finesse, se regarder face à face un impardonnable signe de grossièreté. [...] [A]u Fouta, la duplicité [passait] pour un signe de noblesse et de raffinement » (Monénembo $2008: 60$ ).

On sait que les portraits des princes peuls donnés par l'historiographie coloniale ont généralement été négatifs ne montrant que quelques « roitelets » esclavagistes sans loi ni valeur (Mangolte 1965 : 37-105). C'est d'ailleurs cette image que l'on retrouve chez Ballay, médecin militaire et premier gouverneur français de la Guinée, lorsqu'il évoque Alpha Yaya et les autres princes :

[II] suffira [...] d'enlever leurs captifs, de bouleverser leurs circuits commerciaux et de bâtir nos cités chez les tribus qui leur sont hostiles pour que cette bande d'aristocrates peuls s'évanouisse exactement comme la fumée quand on ouvre grand les portes (Monénembo $2008: 318$ ).

Comme on le voit, Monénembo procède par une mise à distance de la réalité sociologique et politique. Le narrateur s'interdit de présenter un miroir impartial 
de la société peule; il ne vante pas seulement l'esprit chevaleresque des princes, il déplore aussi les intrigues et les guerres internes qui vont affaiblir le royaume et ouvrir la voie à la colonisation. En réalité, Monénembo a l'intuition de ce qui se perd et disparaît de la mémoire collective, et par conséquent, accorde une place à tout ce qui relève du quotidien, des traditions, de l'anecdotique. La distanciation est d'autant plus riche de sens dans le cas d'une réappropriation de l'histoire qu'elle participe de la conservation d'un héritage et de la perpétuation d'une identité culturelle.

Le Terroriste noir, son dixième roman, poursuit cette quête en ressuscitant un tirailleur d'origine guinéenne devenu héros de la Résistance française. Le Terroriste noir est ainsi une un fragment de cette vaste fresque historique :

C'est quand même un héros méconnu, aussi bien ici en France qu'en Guinée. Et puis, de fil en aiguille, je me suis mis à m'y intéresser. Je me suis aussi rendu compte, après avoir écrit Le Roi de Kahel, qu'il complétait un peu Olivier de Sanderval. Ce sont des personnages qui se rencontrent, qui se croisent, qui sont complémentaires. L'un quitte le monde des Blancs, la France, pour aller se mêler à l'histoire des Africains, et l'autre c'est l'inverse : il quitte l'Afrique pour venir se mêler à l'histoire des Blancs en France (Cadet 2012).

Plus que pour Le Roi de Kahel, la fidélité à l'histoire événementielle semble importante ici comme l'explique l'auteur à propos d'Addi Bâ, le personnage principal : "J'ai voulu au maximum que le fil historique soit maintenu et en même temps, je tenais à restituer autant que possible la personnalité d'Addi Bâ, aussi bien la personnalité physique que la personnalité psychologique » (Cadet 2012). Le Terroriste noir tend ainsi à apparaître comme une fiction érudite nourrie de données historiques transposées et remaniés, qui peuvent se prêter à tout un jeu d'allusions et de références à une actualité marquée par les commémorations et la reconnaissance de l'engagement des armées coloniales aux côtés des forces 
françaises. Le récit de Monénembo combine les éléments de la chronique et du témoignage avec le dispositif narratif du roman historique :

[...] c'est un roman. C'est une fiction. Vous savez, moi je suis un romancier. Je ne suis pas un scientifique. Enfin je suis scientifique de formation, mais je ne suis pas un historien au sens scientifique du terme. Je ne suis pas là pour vérifier objectivement les faits. Je suis là pour raconter une histoire. Mais c'est vrai que, en l'occurrence, pour raconter une histoire, quand même on s'inspire des documents. On s'inspire des réalités, des archives en quelque sorte (Cadet 2012).

Ici comme ailleurs, c'est le « conte » qui prime en constituant une prise en charge et une transmission du témoignage par les survivants de la Résistance française. Dès les premières pages, Monénembo multiplie dans Le Terroriste noir les analepses et les prolepses et fait intervenir les témoins, tantôt en convoquant la mémoire du Colonel Melun, tantôt sous le couvert des anecdotes racontées par les autres protagonistes (La Pinéguette, Célestin, Nonon Totor, etc.).

D'une manière plus générale, le récit a été construit avec des matériaux venus d'horizons différents, empruntés aux archives et à la culture populaire. Toutefois, l'auteur non sans quelque habileté fait du fait divers et de la petite histoire la source réelle, le lieu d'un simple rapprochement, réduisant ainsi le fait historique au statut d'indice. En réalité, Monénembo s'amuse à composer une fresque des habitudes et des mentalités de l'époque où dans un petit village des Vosges, les habitants sont confrontés à la présence $\mathrm{d}^{\prime}$ « un nègre, un vrai, aussi noir que s'il se trouvait encore là-bas dans [les] forêts d'Afrique » (Monénembo 2012 : 57). Le Terroriste noir est bien plus proche d'un roman de mœurs de paysans vosgiens précisément documenté que d’un roman historique. L'expérience de la guerre, les intrigues familiales, les attitudes populaires et grossières des personnages sont racontées par la narratrice dans une langue légère et familière. Face aux fictions qui se limiteraient à quelques aménagements des évènements, Monénembo crée une œuvre de témoignage où domine 
l'expression directe et individuelle et où chaque protagoniste partage ses souvenirs. Et comme l'imaginaire de l'auteur est vaste, il se donne la liberté de construire ses intrigues, choisissant dans l'histoire réelle, dans les possibilités de cette rencontre intense et inattendue entre un Bambara et des Vosgiens, les expériences qui le séduisent, soit par leur référence à l'Histoire, soit par leur portée humaine exceptionnelle.

Cette expérience interculturelle fait de ce roman le lieu de déconstruction de préjugés de toute sorte où l'on passe très vite d'un niveau axiologique à un niveau épistémique de la connaissance de l'autre (Todorov 1982). Le Terroriste noir a ainsi une double fonction : d'une part, aiguiser la sensibilité du lecteur sur un chapitre oublié de la Résistance (Monénembo 2012 : 69), d'autre part, divertir avec facilité autour de la vie de «Don Juan » d'Addi Bâ (Monénembo 2012 : 103). Adopté par les habitants de Romaincourt (Vosges) avec lesquels il partageait son quotidien, le tirailleur nègre, «qui refusait la Débâcle et songeait à rejoindre Londres » (Monénembo $2012: 120)$, s'engage avec la Résistance française et en devient l'un des principaux stratèges jusqu'au jour où il est emmené manu militari par les Allemands (Monénembo 2012 : 127). L'engagement de l'auteur pour une réhabilitation du tirailleur nègre s'inscrit dans cette fonction première donnée à l'écriture de l'histoire postcoloniale qui doit « réinterpréter sa propre histoire » (Arend $2008: 24$ ). Le passé n'est pas seulement vu autrement; il s'oppose à une tradition discursive comme une réaction. Face à l'historiographie officielle, Monénembo met en avant la subjectivité de l'écriture de l'Histoire.

\section{Madagascar, 1947: réappropriation de la mémoire et complexification du regard}

Écrit en français et en malagasy, Madagascar, 1947 de Jean-Luc Raharimanana est le témoignage frappant d'une quête intérieure jusque-là inassouvie : «Je rouvre la lettre d'un ami, précieuse pièce dans cette quête de la 
mémoire qui m’obsède » (Raharimanana 2008: 9). Paru six années après son roman Nour (2001), qui reconstruit à partir de la figure de la femme aimée (Nour) le passé de Madagascar des origines jusqu'au moment de la révolte de mars 1947, Madagascar, 1947 est également un texte hybride, un livre-document, une compilation composée d'extraits de témoignages, de photos d'archive, d'extraits de tracts, d'analyses historiques et de commentaires à propos de la rébellion de mars 1947 contre le pouvoir colonial français. L'ouvrage de moins d'une centaine de pages se présente à la fois comme une réflexion et un plaidoyer pour le renouvellement de la relation entre la France et ses anciennes colonies d'Afrique et de l'Océan Indien. Il met l'accent sur la nécessité d'une prise en charge de l'histoire et l'importance de sa transmission aux plus jeunes générations. Pour rompre avec la logique d'une concurrence des mémoires coloniale et postcoloniale, Raharimanana plaide pour une "histoire croisée » au sens où l'entendent Werner et Zimmermann (2003 : 7-36) : «Que raconter du geste du colonisé, ce prétendu vaincu? Comment rendre compte du regret ou même de l'aigreur du colon-ex-colon, ce prétendu vainqueur? »(Raharimanana 2008 : 8-9).

Ébauche plutôt que véritable étude historique, Madagascar, 1947 s'ouvre avec la présentation de Sarin'ny Tahiry Charles Ravoajanahary, historien et grande figure de la lutte pour l'indépendance de Madagascar. En réalité, c'est mû par la volonté de reconquérir une part de vérité historique que Raharimanana dénonce l'absence d'un réel débat sur l'histoire et la mémoire coloniale. Son approche de la question coloniale s'inscrit dans le sens d'une critique idéologique et politique qui invite les nouvelles générations à se «défaire des images d'euxmêmes fabriquées par les métropoles et de construire une autre histoire » (Zytnicki 2003: 9). La pensée de Raharimanana se caractérise par la même préoccupation qui cherche à rétablir la complexité des événements en interrogeant les silences, les non-dits et les mensonges de l'histoire officielle : 
Ma mémoire demande des comptes à la "mère" patrie. Mère patrie ou l'art de maquiller l'invasion brutale et barbare en amour paternel maternel, l'art de requalifier l'agression manifeste en acte de "protectorat" indispensable à ces terres abandonnées [...] » (Raharimanana $2008: 5$ ).

Raharimanana ironise sur le rôle de la mère-patrie qui, au lieu de protéger, détruit des cultures entières, exécute et sème la mort. Le sentiment que l'auteur a de la question mémorielle est empreint de pessimisme et de déception; il est aussi agité et troublé par le déni des classes politiques malgache et française. Il reprouve tout de son temps : les mensonges, les lâchetés, les complaisances d'une ancienne puissance coloniale qui peine à reconnaître l'horreur qu'elle a contribué à créer.

Envisagée par Raharimanana à la fois comme changement de paradigme et contre-discours, cette histoire «autre» est l'aboutissement d'une prise de conscience qui, selon l'historien Didier Nativel, remonte aux années 1970-1980 où «chaque 29 mars, date anniversaire du déclenchement de l'insurrection, est l'occasion d'une introspection collective » (Nativel 2003 : 164). Comme on le voit, cette prise de conscience est relativement récente. Elle vient après de longues années de silence qui ont installé un climat de suspicion et de frustration : " avant 1972, dans cette phase néo-coloniale qualifiée de conservatrice [...], il était difficile de travailler sur les luttes anti-coloniales ou sur l'esclavage. Le refoulement des traumas du passés s'est dans l'ensemble poursuivi » (Nativel 2003 : 158). L'année 1972 est une date charnière dans l'histoire de Madagascar, puisqu'elle marque le début d'un travail mémoriel axé sur le passé et le présent, qu'il s'agisse de l'esclavage pendant la période royale, des luttes anticoloniales ou de la dictature postcoloniale. Dans les représentations théâtrales ${ }^{6}$ de Raharimanana, ses personnages transmettent l'idée d'un être ébranlé dans ses

\footnotetext{
${ }^{6}$ Nous avons eu l'opportunité d'assister à plusieurs représentations de l'auteur notamment à Strasbourg lors du colloque "La relation franco-africaine, une nouvelle histoire politique et littéraire (1975-2015)", Université de Strasbourg, 11-13 Avril 2017.
} 
convictions, troublé par les récits des survivants de la rébellion et marqué par le silence de l'histoire officielle.

Raharimanana refuse le ruminement des souvenirs et exige l'action. Madagascar, 1947 retrace un épisode de l'insurrection de 1947 en intégrant des images d'archives, des rappels historiques et des réflexions sur le devoir de mémoire. Un des épisodes les plus marquants de ce texte est le récit de la visite $\mathrm{du}$ « frère » $\mathrm{du}$ père de l'auteur, un ancien combattant soucieux de transmettre son témoignage. Pour Raharimanana, il est la voix de son temps (Raharimanana 2008 : 25-26). Le «frère » du père, pour reprendre l'expression de l'auteur, a vécu « les sanglants affrontements avec les Français »(Raharimanana 2008 : 25). Les aveux de «l'homme », comme l'appelle l'auteur, en disent long sur la profondeur de la tragédie : «[...] je n'oublierai jamais, je n'oublierai jamais - que Zanahary me pardonne, que les ancêtres épargnent mes enfants, mais ma faute est impardonnable, on ne peut pas faire ça à un être humain... » (Raharimanana 2008 : 26). Tout y est, la violence, l'amertume, le silence. La souffrance de cet ancien combattant qui n'a même plus de nom est concrète et profonde : elle est l'expression spontanée d'une expérience pénible et traumatisante. Et le «silence [qui] a recouvert la maison » (Raharimanana 2008 : 26) ce jour-là s'est répandu à toute la Nation, précise l'auteur. Madagascar, 1947 participe de ce que Didier Nativel a appelé «la complexification du regard porté sur 1947 » (Nativel 2003 : 159) où les bourreaux sont aussi bien malgaches qu'étrangers. Ici, l'écriture de l'histoire cache des enjeux qui dépassent les événements eux-mêmes ; ces enjeux sont d'ordres épistémologiques et idéologiques.

Conscient des enjeux de l'écriture de l'histoire, Raharimanana ouvre son texte en posant la question de la sélection dans l'écriture de l'histoire africaine tout en se demandant au passage si des dates comme 1947 «sont vraiment des dates pertinentes dans l'histoire de l'humanité? Méritent-elles de figurer dans les 
livres d'histoire? » (Raharimanana 2008: 8). La question que l'auteur pose dépasse la situation malgache pour se fondre dans une problématique beaucoup plus générale de l'écriture de l'histoire des subalternes. Sa réponse est un plaidoyer pour « une histoire globale» (Manning 2006) de la violence coloniale en connexion avec la situation postcoloniale. Raharimanana va donc beaucoup plus loin dans ses aspirations et dépasse la temporalité coloniale, puisqu'il évoque les violences et les dérives de la postcolonie (Raharimanana 2008: 33). Comment lire l'histoire des peuples colonisés à partir du débat sur l'écriture de l'histoire coloniale? Telle est la question posée par l'écrivain malgache.

Pour Raharimanana se pose ainsi la question de la « légitimité du Sud de raconter sa propre histoire» (Raharimanana 2008: 25), et de sa capacité à produire un discours scientifique cohérent et crédible sur le passé colonial. Il rejoint ici les préoccupations des historiens qui, à la fin des années 1970, posait déjà la question de l'écriture d'une histoire africaine à partir d'une perspective africaine ${ }^{7}$. Comme Ki-Zerbo en son temps, Raharimanana se pose en défenseur du Sud qui, pour certains, devrait « toujours justifier de sa méthode pour livrer sa version » (Raharimanana 2008 : 25). Réduite au silence jusqu'au début des années 1970, l'historiographie malgache s'est progressivement appropriée une problématique qui touche aussi bien à son identité qu'à sa relation avec l'ancien colonisateur. Pour l'écrivain postcolonial, la question de la sélection se pose en terme de construction d'une «parole historique » légitime, qui tienne compte des voix des victimes car, estime-t-il, «elles ne sont pas audibles [...] non pas parce qu'elles ne veulent pas parler mais parce qu'on ne veut pas les écouter, ce qu'elles racontent dépasse tellement l'entendement qu'on ne peut pas, on ne veut pas y croire » (Raharimanana $2008: 25)$.

\footnotetext{
${ }^{7}$ Ki-Zerbo (1978 : 9). L'historien burkinabè s'insurgeait à l'époque contre la conception hégélienne d'une Afrique sans histoire. Les thèses des historiens de l'époque coloniale comme Coupland, auteur d'une Histoire de l'Afrique Occidentale parue en 1928 ou celles de Charles-André Juliens, Histoire de l'Afrique publiée en 1944, sont battues en brèche.
} 
En racontant l'aventure de son ami Benja, qui bute contre un mur de silence au moment de son enquête à propos des droits des anciens combattants, en relatant les échanges entre son père et un ancien combattant, en citant un extrait du récit Tsiahy publié en 1982 par Koko Jean Marie, un ancien commandant de la guérilla dans le Sud-Est de l'île (Raharimanana 2008 : 29), Raharimanana montre que mars 1947 fait partie de la mémoire individuelle et collective et que cette tragédie devrait trouver sa place dans la mémoire historique. Il confirme ainsi la thèse d'un "profond traumatisme » avancée par Nativel (2003: 159). En même temps, il montre la fragilité de cette «mémoire [qui] reste vivace [dont la] transmission, non assumée par les gouvernements impliqués - de l'Ile comme de France -, est condamnée à la légende et à la rumeur» (Raharimanana 2008: 14) C'est ici qu'il fustige la position des gouvernements postcoloniaux qui, faisant le jeu de l'ancien colonisateur, s'interdise d'évoquer ces pages tragiques de l'histoire coloniale. L'attitude de Marc Ravalomanana «prônant l'oubli» (Raharimanana 2008: 16) est symptomatique des gouvernements postcoloniaux soucieux de préserver leurs intérêts et «les "bonnes relations" avec la France» (Raharimanana 2008 : 16). L'écrivain note que :

Là réside le problème des pays africains quand il s'agit d'évoquer les massacres coloniaux ou tout simplement les préjudices de la colonisation. Crainte qu'en revenant sur le passé, ils n'irritent pas la France et ses gouvernants et que ces derniers, en représailles, ne leur coupent les vivres... (Raharimanana $2008: 16$ ).

Cette posture va même à l'encontre d'un sentiment largement partagé par l'ensemble du corps social et d'une prise de conscience de l'ancienne puissance coloniale qui y voit une «des périodes sombres de l'histoire commune ${ }^{8}$

\footnotetext{
${ }^{8}$ Ces propos de Jacques Chirac en visite à Madagascar le 21 juillet 2005 sont rapportés par l'auteur. Le président français évoquait à l'époque «le caractère inacceptable des répressions » et la nécessité d'un « travail de mémoire » (Raharimanana $2008: 15$ ).
} 
(Raharimanana 2008 : 15). La position plutôt conciliante de Marc Ravalomanana, chef de l'Etat malgache au moment de la visite du président Chirac en juillet 2005, tranche avec la prise de conscience chez la plupart des intellectuels du pays. Elle révèle la complexité de la question mémorielle parce qu'elle implique des enjeux identitaires et politiques. En d'autres termes, les revendications postcoloniales ne s'adressent pas uniquement à l'Occident, mais aussi aux autorités politiques et à une aristocratie locale, souvent complices des exactions coloniales.

\section{Conclusion}

Nous avons ouvert cette réflexion sur l'écriture de l'Histoire avec l'idée de « rendre l'Histoire conte » émise par Thierno Monénembo. L'Histoire ne rend pas seulement compte en ce sens que l'historiographie favorise un retour sur les événements $\mathrm{du}$ passé, une reconstruction du passé de manière objective, exhaustive et impartiale, mais elle permet aussi de rendre conte, c'est-à-dire de mettre en avant le dire, la narration et la textualité de l'Histoire. Rendre conte relève la dimension de l'oralité, d'un récit se disant. Cette idée renforce le rôle du narrateur qui organise le récit mais aussi celui de l'auteur comme conteur qui fait appel à une mémoire sélective et parfois défaillante. Le Roi de Kahel et Le Terroriste noir ont été construits selon le même principe d'une fictionnalisation d'une histoire individuelle et collective véridique. Les besoins d'une réécriture de l'histoire coloniale contenaient plus que le simple désir de varier artificiellement la représentation des faits et des événements; ils s'allient à une soif d'imposer sa propre vision du monde, où l'éveil de l'imagination se relie à celui de l'affirmation de sa différence. Puisqu'il ne s'agit pas pour l'auteur d'écrire une histoire scientifique, il puise dans la tradition populaire de la mémoire collective tout en gardant, par bribes, le souci d'exactitude de l'historiographie. 
Comme Monénembo, Raharimanana s'inscrit dans un processus où la recherche archivistique est au fondement de l'écriture littéraire. Ce processus est marqué par deux logiques apparemment contradictoires mais fortement liés. La première logique est l'importance du travail mémoriel qui passe par une reconstruction aussi exacte que possible de l'Histoire de la rencontre entre l'Europe et l'Afrique. Or ce travail ne peut se faire qu'à partir des archives occidentales qui racontent l'Histoire selon ses propres modalités. La seconde logique est constitutive du statut de l'écrivain qui n'entend pas faire œuvre d'historien, mais souhaite contribuer à la construction d'une mémoire autochtone à partir d'une écriture à la fois référentielle et fictionnelle. Comme nous l'avons vu avec Monénembo et Raharimanana, il s'agit d'une recherche ingénieuse et d'une exigence constante d'originalité. Mais pour rendre conte il faut également savoir distraire, instruire stimuler et rafraîchir sans cesse l'attention du lecteur, ne rien couler dans les moules tout prêt de la pensée ni de l'expression.

\section{Bibliographie}

Arend, E. (2008). «Histoire, littérature et l'écriture de l'histoire ». Dans E. Arend, D. Reichardt et E. Richter. (dir.), Histoires inventées. La représentation du passé et de l'histoire dans les littérature française et francophones. Francfort, Peter Lang, p. 15-32.

Boni, N. (1962). Le crépuscule des temps anciens. Paris, Présence africaine.

Botte, R. (1990). «Pouvoir du Livre, pouvoir des hommes : la religion comme critère de distinction ». Journal des africanistes, tome 60, fascicule 2, p. 37-51. 
Boulègue, J. (1981). «Un empire peul dans le Soudan occidental au début du XVII siècle». Dans 2000 ans d'histoire africaine. Le sol, la parole et l'écrit. Mélanges en hommage à Raymond Mauny, tome II. Paris, Société française d'histoire d'outre-mer, p. 699-706.

Cadet, J.-F. (2012). «"Le Terroriste noir" de Tierno Monénembo : "Je suis là pour raconter une histoire" » (entretien). RFI [en ligne], 25 septembre ; http://www.rfi.fr/france/20120925-le-terroriste-noir-tierno-monenembo-jesuis-raconter-une-histoire [consulté le 26.11.2019].

Chalaye, S. (2002). Nègres en images. Paris, L'Harmattan.

Chanda, T. (2008). «Tierno Monénembo revisite la colonisation » (entretien). Jeune Afrique [en ligne], 2 juin; https://www.jeuneafrique.com/132568/culture/tierno-mon-nembo-revisitela-colonisation/ [consulté le 06.07.2019]

De Meyer, B. (2011). «La colonisation selon Sanderval : Le Roi de Kahel de Tierno Monénembo ». French Studies in Southern Africa, n41, p. 60-79.

Fèvre, A. (2017). «Tierno Monénembo : "J'ai eu envie de raconter l'histoire de la Guinée indépendante" »(entretien). Le Point [en ligne], 27 décembre; http://afrique.lepoint.fr/culture/tierno-monenembo-j-ai-eu-envie-deraconter-1-histoire-de-la-guinee-independante-27-12-20172182624_2256.php. [consulté le 31.01.2019].

Imorou, A. (2010). "L'étranger, le frère, l'ami: variations autour du roi de Kahel », InterFrancophonies, n³, p. 1-13.

Ki-Zerbo, J. (1978). Histoire de l'Afrique noire, d'hier à demain. Paris, Hatier.

Kohlhauer, M. (2011). «Écrire l'Histoire, la part de l'art». Dans M. Kohlhauer (dir.), Fiction de l'Histoire. Écriture et représentations de l'Histoire dans la littérature et les arts. Chambery, Éditions de l'Université Savoie Mont Blanc, p. 21-57.

Liger, B. (2010). «Avec les Crapauds-brousse, Monénembo s'emploie à livrer une chronique sociale acerbe de la société africaine». L'Express [en ligne], 6 
août; https://www.lexpress.fr/culture/livre/les-crapaudsbrousse_910050.html [consulté 04.11.2019].

Malonga, J. (1959). La légende de M’Pfoumou Ma Mazono. Paris, Présence africaine. Mangolte, J. (1968). «Le chemin de fer de Konakry au Niger (1890-1914)». Revue française d'histoire d'outre-mer, t. 55, n 198, p. 37-105.

Manning, P. (2006). World History. Global and Local Interactions. Princeton, Markus Wiener Publishers.

Mbembe, A. (2013). Sortir de la grande nuit. Essai sur l'Afrique décolonisée. Paris, La Découverte.

Migraine-George, T. (2013). From Francophone to World Literature in French. Ethics, Poetics, E Politics. Lincoln et Londres, University of Nebraska Press.

Mofolo, T. (2010 [1940]). Chaka, Paris, Gallimard.

Monénembo, T. (2012). Le terroriste noir. Paris, Editions du Seuil.

Monénembo, T. (2008). Le Roi de Kahel. Paris, Editions du Seuil.

Nativel, D. (2003). "L'historien et le défi des mémoires à Madagascar. Construction de l'objet et demande sociale». Dans S. Dulucq et C. Zytnicki (dir.), Décoloniser l'histoire? De "l'histoire coloniale » aux histoires nationales en Amérique latine et en Afrique (XIX ${ }^{e}-X X^{e}$ siècles). Paris, Publication de la Société Française d'Histoire d'Outre-Mer, p. 151-167.

Ngandu Nkashama, P. (1999). Mémoire et écriture de l'histoire dans «Les Ecailles du Ciel » de Tierno Monénembo. Paris, L'Harmattan.

Paravy, F. (2014). «De l'archive au roman, ou les enjeux d'une réécriture : Le Roi de Kahel de Tierno Monénembo ». Amnis [en ligne], n 13.

Raharimanana, J.-L. (2008). Madagascar, 1947, essai, Paris, Vents d'ailleurs., éd. bilingue.

Raharimanana, J.-L. (2001). Nour, 1947. Paris, Le Serpent à Plumes.

Rancière, J. (2012). Figures de l'histoire. Paris, PUF. 
Rüsen, J. (1982). «Formen des historischen Erzählens ». Dans R. Koselleck, H. Lutz et J. Rüsen (dir.): Formen der Geschichtsschreibung. Munich, DTV.

Semujanga, J. (2004). «La mémoire transculturelle comme fondement du sujet africain chez Mudimbe et Ngal ». Tangence, $\mathrm{n}^{\circ}$ 75, p. 15-39.

Smith, A. (2006). « Migrance, hybridité et études littéraires postcoloniales ». Dans N. Lazarus (dir.), Penser le postcolonial. Une introduction critique. Paris, éditions Amsterdam, p. 359-385.

Socé, O. (1962). Contes et légendes d'Afrique noire. Paris, Nouvelles Editions latines.

Tamsir Niane, D. (1960). Soundjata ou l'épopée mandingue. Paris, Présence africaine.

Werner, M. et B. Zimmermann. (2003). « Penser l'histoire croisée : entre empirie et réflexivité ». Annales HSS, n 58-1, p. 7-36.

Zytnicki, C. (2003). «'La maison, les écuries. L'émergence de l’histoire coloniale en France (des années 1880 aux années 1930)». Dans S. Dulucq et C. Zytnicki, Décoloniser l'histoire? De «l'histoire coloniale» aux histoires nationales en Amérique latine et en Afrique (XIXe-XX ${ }^{e}$ siècles). Paris, Publication de la Société Française d'Histoire d'Outre-Mer, p. 9-23. 